\title{
Atomic Scale Composition Profiling of Ferroelectrics via Laser-Pulsed Atom Probe Tomography and Cross-correlative Transmission Electron Microscopy
}

\author{
Rita Kirchhofer ${ }^{1}$, David R. Diercks ${ }^{1}$, Brian P. Gorman ${ }^{1}$, Geoff Brennecka ${ }^{2}$ \\ ${ }^{1 .}$ Metallurgical and Materials Engineering, Colorado School of Mines, Golden, CO. \\ ${ }^{2}$ Sandia National Laboratories, Albuquerque, NM
}

Ferroelectric oxides are used in a wide variety of applications including capacitors, transistors, piezoelectric transducers, and RAM devices. The perovskite family has proven to be especially useful, with materials such as lead zirconium titanate (PZT) and barium titanate (BT) becoming the industry standards in dielectric and multiferroic applications. Through substitutions of niobium or lanthanum for some of the lead, PNZT and PLZT relaxor ferroelectrics are created. They have extraordinarily high piezoelectric and electrostrictive coefficients, respectively making them useful in electromechanical applications. Tuning the dielectric and piezoelectric properties of these materials can be achieved through compositional tuning near the morphotropic phase boundaries. It has been proposed that relaxor ferroelectrics achieve their electrostrictive properties through nanoscale phase separation, although further atomic scale chemical and structural characterization is required. Atom probe tomography (APT) is capable of analyzing the atomic scale, 3-D distribution of atoms in a solid; however, to date this technique has not been applied thoroughly to ferroelectric oxides.

In order to fully understand the field evaporation characteristics of PZT and its related compounds, laser-assisted APT coupled with transmission electron microscopy (TEM) pre- and post-APT analysis was performed on several different ferroelectric oxides. Samples consisted of bulk sintered pellets as well as thin films deposited on metallic substrates. Specimens were prepared from these samples using standard in-situ FIB liftout techniques. Specimen radii and shank angles were varied in order to study the effects of thermal pulsing on these materials. APT instrumental parameters such as laser pulse energy, acquisition rate, and specimen base temperature were optimized to obtain accurate cation and anion stoichiometry of the oxides. A representative mass spectrum for 53/47 PZT is shown in Figure 1 under acquisition conditions that yielded close to the correct stoichiometry (shown in the inset table). The changes in the $\mathrm{Pb}$ and $\mathrm{Zr}$, or $\mathrm{Pb}, \mathrm{Nb}$ and $\mathrm{La}$, content could be correlated to segregation of species within the material. The combination of the APT and TEM techniques allowed for crystal structure analysis and accurate reconstructions of the atom probe data via quantification of the evaporation field as shown in Figure 2. The piezoelectric response of these specimens to the high electric fields in the atom probe can have a significant effect on the quality of the data achieved $[1,2]$.

[1] This research is supported by NSF Award Number 1040456.

[2] Sandia National Laboratories is a multi-program laboratory managed and operated by Sandia Corporation, a wholly owned subsidiary of Lockheed Martin Corporation, for the U.S. Department of Energy's National Nuclear Security Administration under contract DE-AC04-94AL85000. 


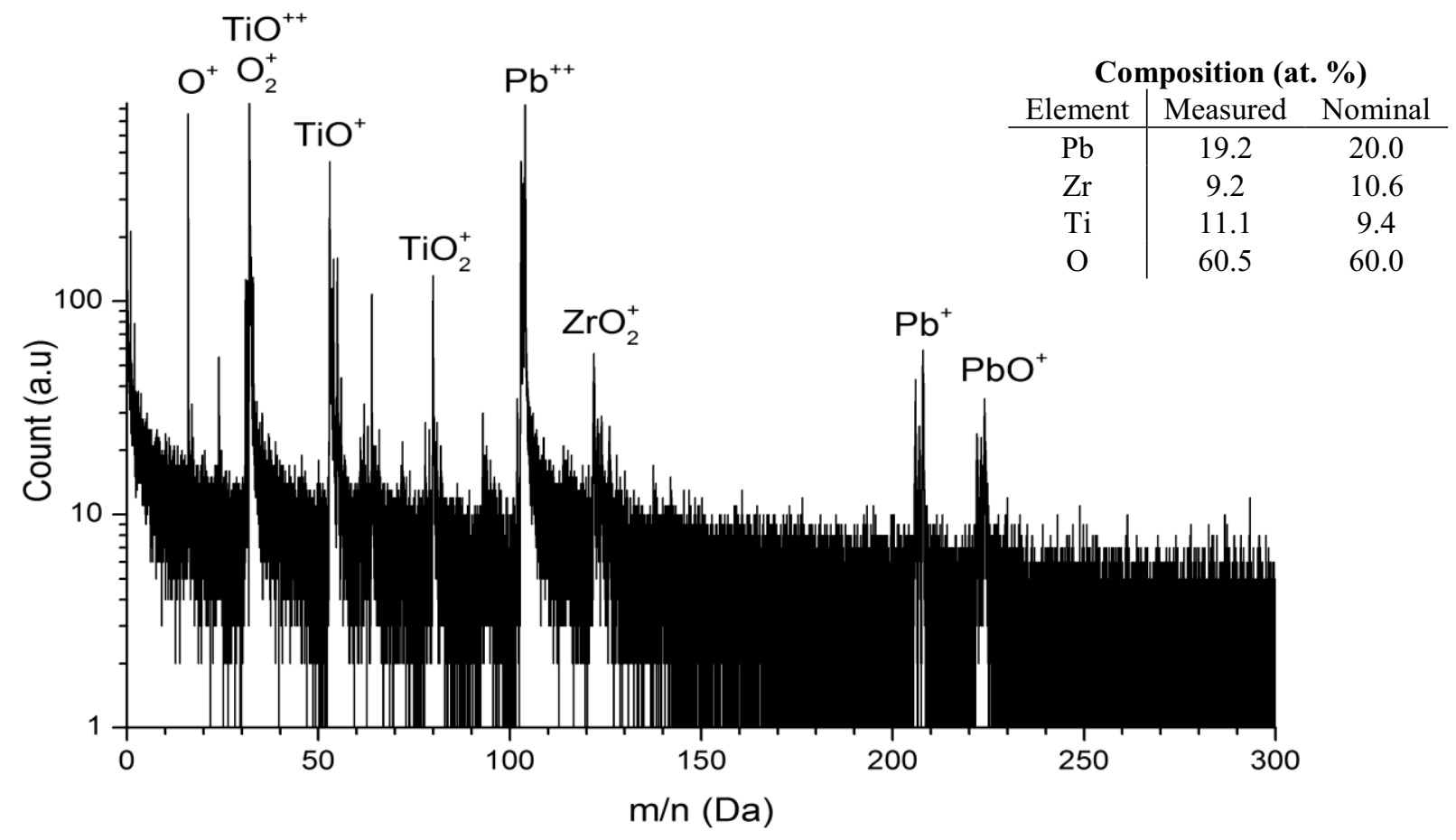

Figure 1. Mass spectrum for 53/47 PZT material and corresponding composition (inset table) obtained via APT under acquisition conditions that yielded the correct composition of the oxide.

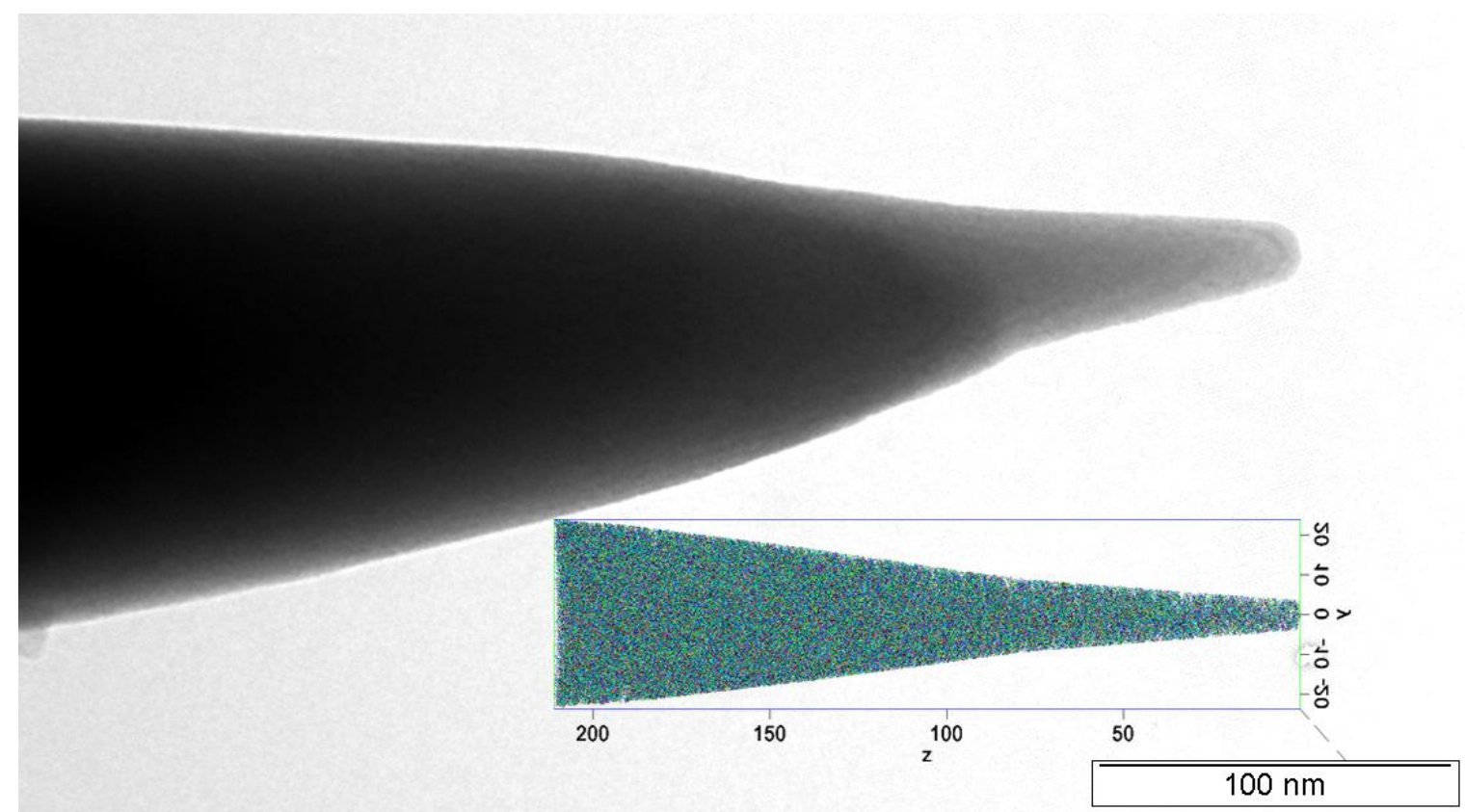

Figure 2. Transmission electron micrograph of a bulk PZT specimen analyzed via APT, and the corresponding reconstruction (inset) shown at the same scale. 